\title{
Soft X-ray Characterization of Block Copolymers for Directed Self Assembly
}

\author{
R. Joseph Kline and Daniel F. Sunday \\ National Institute of Standards and Technology, 100 Bureau Drive, Gaithersburg, MD 20899, \\ joe.kline@nist.gov
}

The microelectronics industry continues to shrink the size of the electronic devices as they follow Moore's Law. They have reached the fundamental limits of current photolithography technology. Current nanodevices require multipatterning methods in addition to photolithography to pattern the sub- $40 \mathrm{~nm}$ pitch structures in current products. One new method being investigated by the industry is called directed self assembly (DSA). DSA is a combination of bottom-up and top-down patterning. The synthetic chemistry of a block copolymer (BCP) determines the feature size and conventional photolithography patterns a chemical template that guides the BCP structure to put the structure in the right place. The template is an integer multiple of the natural spacing of the BCP, and the BCP epitaxially arranges on the template to form the final pattern. The industry is evaluating both cylinder and lamella forming BCPs.

Vertical cylinders can be used for forming or "shrinking" contact holes. Lamella can be used to form a large number of parallel lines for patterning a large grating structure such as finFETs or metal lines. Both of the structures have a number of critical metrology challenges. The threedimensional (3D) structure of these $<25 \mathrm{~nm}$ nanostructures of multiple polymers determine the performance of the patterning technology. Additionally, the sharpness of the interface between the two polymer phases determine how sharp of a pattern can be transferred to the integrated circuit during nanofabrication. We will report on metrology methods we have developed using soft X-rays and small angle X-ray scattering (SAXS) to characterize both the 3D shape and the interfacial width between the polymer blocks.[1-3] These measurements provide critical insight to the microelectronics industry.

\section{References}

[1] Sunday, D.F. et al. (2015) J. Appl. Cryst. 48 (5), 1355-1363

[2] Sunday, D.F. et al. (2019) J. Appl. Cryst. 52 (1), 106-114

[3] Sunday, D.F. et al. (2018) Macromolecules 51 (1), 173-180 